\title{
Adherence to subcutaneous interferon beta-la treatment using an electronic injection device: a prospective open-label Scandinavian noninterventional study (the ScanSmart study)
}

This article was published in the following Dove Press journal:

Patient Preference and Adherence

\author{
Elena Didenko Pedersen ${ }^{1,2}$ \\ Egon Stenager 3,4 \\ JL Vadgaard ${ }^{5}$ \\ MB Jensen ${ }^{6}$ \\ R Schmid ${ }^{7}$ \\ N Meland ${ }^{8}$ \\ G Magnussen? \\ Jette L Frederiksen ${ }^{10,11}$ \\ 'Akershus University Hospital, \\ Department of Neurology, \\ Loerenskog, Norway; ${ }^{2}$ Sandvika \\ Nevrosenter, Sandvika, Norway; \\ ${ }^{3} \mathrm{MS}$ Clinic of Southern Jutland, \\ Department of Neurology, Hospital \\ of Southern Denmark, Odense, \\ Denmark; ${ }^{4}$ Institute of Regional \\ Research, University of Southern \\ Denmark, Odense, Denmark; \\ ${ }^{5}$ Department of Neurology, Sjællands \\ University Hospital, Roskilde \\ NeuroCenter, Roskilde, Denmark; \\ ${ }^{6}$ Department of Neurology, \\ Nordsjaellands Hospital, Hilleroed, \\ Denmark; ${ }^{7}$ MS Clinic, Department \\ of Neurology, Vejle Hospital, Vejle, \\ Denmark; ${ }^{8}$ Smerud Medical Research, \\ Oslo, Norway; ${ }^{9}$ Merck Norway, An \\ Affiliate of Merck AB (Merck AB \\ NUF), Oslo, Norway; ${ }^{10}$ Department \\ of Neurology, Rigshospitalet Glostrup, \\ Faculty of Health and Medical Science, \\ Glostrup, Denmark; "Institute of \\ Clinical Medicine, Copenhagen \\ University, Copenhagen, Denmark
}

Correspondence: Elena Didenko Pedersen

Sandvika Nevrosenter, Sandviksveien I78, N-I 337 Sandvika, Norway

Tel +47924 I I I4 I

Email elena.d.pedersen@outlook.com
Background: Disease modifying drugs help control the course of relapsing remitting multiple sclerosis (RRMS); however, good adherence is needed for long-term outcomes.

Objective: To evaluate patient adherence to treatment with subcutaneous interferon beta-1a using RebiSmart ${ }^{\mathbb{B}}$ and assess injection-site reactions and treatment satisfaction.

Methods: This prospective, single-arm, open-label, noninterventional multicenter Phase IV trial included disease modifying drug-experienced mobile patients with RRMS. Adherence was measured over 12 weeks. Items 13-23, 35, 37, and 38 of the Multiple Sclerosis Treatment Concerns Questionnaire (injection-site reactions and treatment satisfaction) were recorded at 12 weeks.

Results: Sixty patients were recruited (mean age 43.7 [ \pm SD 7.9] years; $83 \%$ female; mean years since multiple sclerosis diagnosis 6.7 [SD 4.5]). Adherence data were obtained in 54 patients only due to technical problems with six devices. Over 12 weeks, $89 \%(n=48)$ of patients had $\geq 90 \%$ adherence to treatment. Most patients experienced mild influenza-like symptoms and injectionsite reactions, and global side effects were minimal. Most patients (78\%) rated the convenience as the most important aspect of the device, and most experienced no or mild pain.

Conclusion: RRMS patients treated with subcutaneous interferon beta-1a, administered with RebiSmart, demonstrated generally good adherence, and the treatment was generally well tolerated.

Keywords: adherence, multiple sclerosis, relapsing remitting MS, subcutaneous interferon beta-1a, RebiSmart, trial

\section{Introduction}

Multiple sclerosis (MS) is a chronic neurological disease mediated by an inflammatory process within the central nervous system and is the leading cause of disability among young adults. ${ }^{1}$ The exact cause of MS is unknown, although an autoimmune process has been implicated. Genetic susceptibility also has a role in disease initiation, ${ }^{2}$ in addition to, as yet unidentified, environmental factors. ${ }^{3}$

There is no cure for MS, but disease modifying drugs (DMDs) that can reduce relapse frequency and slow disability progression are available. ${ }^{4}$ Interferon (IFN) beta is a first-line DMD for relapsing remitting multiple sclerosis (RRMS). Its efficacy has been proved in several randomized controlled trials. ${ }^{5}$ However, the clinical and economic benefits of treatment with IFN beta, as with other DMDs, are dependent on good adherence to treatment..$^{6-8}$ 
Adherence is defined by the World Health Organization as "the extent to which a person's behavior - taking medication, following a diet, and/or executing lifestyle changes - corresponds with agreed recommendations from health care providers." There are two main components to evaluating adherence: the maintenance of a drug regimen over time (persistence) and taking the medication according to the prescribed dose and schedule (compliance). As with treatments for other chronic diseases, low adherence to IFN beta treatment has been reported. ${ }^{10}$

Common side effects to IFN beta treatment, such as injection-site reactions and influenza-like symptoms, are known to lead to low adherence. ${ }^{10}$ Therefore, the implementation of measures to reduce these side effects may help to improve adherence to IFN beta treatment.

The use of an electronic injection device can reduce the severity of injection-site reactions and improve compliance of MS patients with IFN beta treatment. ${ }^{11-13}$ The RebiSmart ${ }^{\circledR}$ device, an electronic/electromechanical autoinjector, was designed to improve patient satisfaction when administering subcutaneous (sc) IFN beta-1a for the treatment of RRMS. The device enables patients to personalize the injection attributes (injection depth, speed, and duration), track their injection history, and provides patients and physicians with a tool to monitor patient adherence to subcutaneous interferon (sc IFN) beta-1a.

The aim of this study was to evaluate adherence to treatment with sc IFN beta-1a among patients with RRMS using the RebiSmart device over 12 weeks. The secondary objectives were to assess patient satisfaction and experience of factors that contribute to adherence.

\section{Methods}

\section{Research ethics and patient consent}

Due to the nature of the study, the Norwegian (REK sør-øst B) and Danish (Videnskabsetiske komite Region Syddanmark) Regional Ethics Committees concluded that no approval was required. All patients provided written informed consent to participate in the study before any study-related activities were carried out.

\section{Study design}

The ScanSmart study was a prospective, noninterventional observational Phase IV trial among patients with RRMS. One clinic in Norway and five clinics in Denmark participated in this study. The study is registered on ClinicalTrials.gov (NCT01125475).

Inclusion criteria were as follows: males and females aged between 18 and 65 years old, RRMS according to the revised McDonald Criteria (2005), an expanded disability status scale score (EDSS) of $<6$ at the screening visit, stable treatment (defined as treatment after a titration period) with sc IFN beta-1a 22/44 $\mu \mathrm{g}$ administered subcutaneously three times per week for a minimum of 4 weeks prior to inclusion using the RebiSmart electronic/electromechanical autoinjector, and previous treatment with DMDs for a minimum of 6 months prior to the screening visit. Female patients were neither pregnant nor breastfeeding and were either postmenopausal or surgically sterile or using a highly effective method of contraception for the duration of the study.

Patients were excluded from this trial if they had a relapse within 30 days prior to the first visit, had any contraindications to treatment with IFN beta-1a, or had any other significant diseases that could influence the treatment.

\section{Visits}

Patients attended two scheduled visits: at screening or baseline (day 0) and after 12 weeks or at the end of the treatment. Data obtained from the RebiSmart device were registered electronically at day 0 (visit 1 ) and after 12 weeks. Disease status (EDSS) was recorded in the case report form at the first visit. At the 12th week visit, the Multiple Sclerosis Treatment Concerns Questionnaire (MSTCQ) was distributed to the patients. The patients used a patient diary to register reasons for missed injections.

\section{Treatment}

RebiSmart administers sc IFN beta-1a from multidose cartridges. The device is designed to inject a fixed volume of $0.50 \mathrm{~mL}$ per injection. Each multidose cartridge was prefilled with $1.5 \mathrm{~mL}$ of solution containing 66/132 $\mu \mathrm{g}$ IFN beta-1a. The cartridge was designed to deliver three individual doses of $0.5 \mathrm{~mL}$ solution for injection containing 22 or $44 \mu \mathrm{g} \mathrm{sc}$ IFN beta-1a, as per label and normal clinical practice. Treatment was given for a period of 12 weeks after baseline and injections were administered at the same time of day on the same three days of the week, with at least 48 hours between each administration.

\section{Primary endpoint}

The primary endpoint was adherence to treatment in patients with RRMS using the RebiSmart device to self-inject sc IFN beta-1a in a multidose cartridge over 12 weeks.

\section{Secondary endpoints}

The secondary endpoints were the occurrence of injectionsite reactions and patient satisfaction, determined from the MSTCQ. The MSTCQ is a 38-item questionnaire validated 
to identify patient concerns with treatment with IFN beta; each item has a choice of five responses, except for item-38, which had a choice of six responses. We assessed patients' responses on the injection-system satisfaction domain (items 13-23): items 13-16, influenza-like symptoms; items 17-20, injection-site reactions; and items 21-23, global side effects; and 35, benefit; and 37, 38, pain-rating scale and pain-rating grade from the patient questionnaire at the end of 12 weeks.

In the MSTCQ form used in the study, the individual scores for items 13-23 were from 1 to 5 . The scores for items 13-20 were reverse coded, so that a higher score indicated a greater negative impact. The overall score for items 21-23 ranged from 3 to 15 , with the highest scores indicating the worst possible treatment satisfaction. The patient response to pain in the past 4 weeks was measured using a $100 \mathrm{~mm}$ visual analog scale, in which higher scores indicated greater pain. Reasons for missed injections were recorded in the patient treatment diary.

\section{Statistical analyses}

No statistical hypotheses were planned, as this was a singlearm study. The planned sample size of 100 patients is based on the estimate that $90 \%$ of patients treated for 12 weeks will be $80 \%$ adherent to treatment. This produces a $95 \%$ confidence interval equal to the sample proportion plus or minus $5 \%-6 \%$ when the estimated proportion is $90 \%$.

The analysis of the primary endpoint was descriptive, with the proportion of patients with adherence of $\geq 100 \%$, $\geq 95 \%, \geq 90 \%, \geq 85 \%, \geq 80 \%$, and $\geq 75 \%$ to sc IFN beta-1a three times a week administered with the RebiSmart. Patients at the lower cutoff of $75 \%$ were regarded as truly nonadherent. Exact 95\% CI was calculated using the Clopper-Pearson method.

Adherence was calculated as the number of injections administered, multiplied by 100 and divided by the expected number of injections (36 injections [100\% adherence]). If treatment was terminated prematurely, the expected number of injections was calculated from the time on treatment in weeks, multiplied by the number of weeks on treatment. If a patient was lost to follow-up, no attempt was made to impute the adherence for this patient. If a RebiSmart device was lost or damaged, the patient was rescreened. If a patient completed the study but no injection data could be obtained from RebiSmart, the patient was excluded from the analysis. If RebiSmart data were lost for part of the treatment period two estimates of adherence were planned. In the first estimate, it would be assumed that no injections were made in the part of the treatment period where the data were lost; in the second estimate, the days for which the data were lost would be excluded.

Assessment of factors contributing to adherence were analyzed on the proportion of patients with $\geq 80 \%$ and $\geq 100 \%$ adherence using logistic regression with demographic and safety variables (age, age at diagnosis, gender, disease duration, employment status, education, marital status, diseasemodifying therapy use, number of relapses, EDSS score at visit 1 , influenza-like symptoms, injection-site reactions, global side effects, benefits of treatment, pain, dose, and number of relapses in the 2 years before screening) as covariates. Covariates significant on a 5\% level in the univariable logistic regression were planned to be included in the final multivariate models.

Analyses of the secondary endpoints were also descriptive only. The secondary endpoints comprised the MSTCQ scores $( \pm \mathrm{SD})$ of the items measured.

Data analyses were performed using $\mathrm{SAS}^{\circledR}$ software, version 9.2 (Cary, NC, USA), after importing data from a VieDoc ${ }^{\mathrm{TM}}$ database. All patient data were presented in separate listings. All analyses were based on data pooled across clinics.

\section{Results}

\section{Patients}

Due to recruitment difficulties, and in order to finalize the trial within reasonable timelines, the trial was stopped when a total of 60 subjects were screened and gave signed informed consent to participate in the study. Demographic characteristics of the study population are presented in Table 1. MS history is shown in Table 2, and EDSS score at baseline is shown in Figure 1. There were no withdrawals from the study.

One patient was included in the study despite not having stable RebiSmart treatment in the last 4 weeks before baseline. For six patients, all RebiSmart data were lost owing to the data not being recorded in the electronic case report form and, in accordance with the study protocol, these patients were not included in the calculation of the primary endpoint. Of these patients, one patient recorded one injection-site reaction as an adverse drug reaction; one patient recorded 15 missed injections due to pain at injection site; one patient forgot to take one injection; and two patients also reported ongoing injection-site pain at the time of the last visit. For one additional patient, the RebiSmart data for the days between the baseline and visit 2 were lost due to a site error.

\section{Primary endpoint}

A total of 54 patients were included in the calculation of the primary efficacy endpoint. Of these patients, $51(94 \%)$ 
Table I Patient demographics

\begin{tabular}{|c|c|c|c|c|c|c|c|}
\hline & \multicolumn{6}{|l|}{ Study center } & \multirow[t]{2}{*}{ Total } \\
\hline & Sønderberg & Vejle & Roskilde & Glostrup & Hillerød & Sandvika & \\
\hline Patients, $\mathrm{n}$ & 10 & 1 & 10 & 26 & 8 & 5 & 60 \\
\hline Female, n (\%) & $8(80)$ & I $(100)$ & $9(90)$ & $23(88)$ & $6(75)$ & $3(60)$ & $50(83)$ \\
\hline Age in years, mean (SD) & $42.0(5.0)$ & 47.4 (NA) & $45.7(6.0)$ & $44.6(9.1)$ & $41.8(8.3)$ & $40.3(10.0)$ & $43.7(7.9)$ \\
\hline \multicolumn{8}{|l|}{ Employment status, n (\%) } \\
\hline Full time & $3(30)$ & $0(0)$ & $0(0)$ & $7(27)$ & I (I3) & $3(60)$ & $14(23)$ \\
\hline Part time & $5(50)$ & I $(100)$ & $4(40)$ & $10(38)$ & $4(50)$ & I (20) & $25(42)$ \\
\hline Unemployed & $2(20)$ & $0(0)$ & $6(60)$ & $9(35)$ & $3(38)$ & I (20) & $21(35)$ \\
\hline \multicolumn{8}{|l|}{ Education status, n (\%) } \\
\hline Elementary & $3(30)$ & $0(0)$ & $4(40)$ & $2(8)$ & $0(0)$ & $0(0)$ & $9(15)$ \\
\hline Intermediate & $6(60)$ & $0(0)$ & $6(60)$ & $18(69)$ & $7(87.5)$ & $3(60)$ & $40(67)$ \\
\hline Higher & $\mathrm{I}(\mathrm{I0})$ & I $(100)$ & $0(0)$ & $6(23)$ & I (I2.5) & $2(40)$ & $11(18)$ \\
\hline \multicolumn{8}{|l|}{ Marital status, n (\%) } \\
\hline Single/divorced & $5(50)$ & I $(100)$ & $I(10)$ & $5(19)$ & $I(13)$ & I (20) & $14(23)$ \\
\hline Married/living with partner & $5(50)$ & $0(0)$ & $9(90)$ & $2 \mathrm{I}(8 \mathrm{I})$ & $7(87)$ & $4(80)$ & $46(77)$ \\
\hline
\end{tabular}

had adherence $\geq 75 \%$ (95\% exact [Clopper-Pearson] CI: $85 \%-99 \%)$. Thirty-four (63\%) patients had adherence of $\geq 100 \%$. Only three ( $6 \%$ ) patients had adherence $<75 \%$ and could be defined as nonadherent according to the study protocol (Table 3). The proportion of patients at each level of adherence is shown in Figure 2. The reasons for missed injections were recorded in the patient diaries only at the centers in Denmark. Fifteen patients (27\%) reported missing an injection. The most common patient-reported reason for missing an injection was forgetting to take the injection (11 patients $[20 \%])$.

\section{Secondary endpoints}

The mean ( \pm SD) MSTCQ subscale scores were 10.3 (3.6) for injection-site reactions and 7.4 (3.9) for influenza-like symptoms, out of a maximum of 20 for the worst possible outcomes, indicating that these side effects were mild. The global side effects subscale had a mean (SD) value of 5.0 (2.4), and all of the items in the global side effects subscale had a mean value $<2$, out of a maximum of 15 for the worst possible outcome, indicating the best possible outcome for these assessments. The majority of patients (47 of $60[78 \%]$ ) stated that the overall convenience was

Table 2 MS history

\begin{tabular}{ll}
\hline & $\begin{array}{l}\text { Mean value } \\
( \pm \mathbf{S D}), \text { years }\end{array}$ \\
\hline Age at the time of first attack & $34.5(8.1)$ \\
Time since first attack & $9.1(5.8)$ \\
Age at the time of MS diagnosis & $37.0(7.6)$ \\
Time since MS diagnosis & $6.7(4.5)$ \\
Time since last clinical relapse & $4.0(3.3)$ \\
\hline
\end{tabular}

Abbreviation: MS, multiple sclerosis. the most important benefit of the injection system used in the study. The remainder rated fewer injection-site reactions (2 patients [3\%]), less injection pain (7 patients [12\%]), fewer influenza-like symptoms ( 1 patient [2\%]) and fewer physical side effects ( 3 patients [5\%]) as the most important benefits. Regression analysis did not identify any factors associated with $\geq 80 \%$ or $\geq 100 \%$ adherence.

The mean $( \pm \mathrm{SD})$ MSTCQ score for pain (item 37) was 25.3 (24.7), indicating that pain during the last 4 weeks was at the lower end of the scale. The mean (SD) pain score recorded during the last 4 weeks of the study was 1.9 (1.0), indicating that most of the patients had either no pain or mild pain from the injections, and no patient had a score higher than 4 (Distressing).

\section{Discussion}

Poor medication adherence is a major problem in the treatment of MS. The Global Adherence project ${ }^{14}$ reported that in 2,566 patients enrolled at 176 sites in 22 countries, overall, 25\% of patients taking DMDs for MS were not adherent (defined as missing at least one dose in the last 4 weeks) to their prescribed treatment regimen (for sc IFN beta-1a, $73 \%$ of patients were nonadherent to their therapy). A review of patient adherence to DMDs reported adherence rates ranging from $41 \%$ to $88 \%$ regardless of the definition of adherence used, type of DMD agent, or study design. ${ }^{7}$ Similar results were reported in a survey of 709 patients recruited from academic and community MS clinics in the USA, which showed that, overall, 37\% of respondents were considered nonadherent (defined as missing $\geq 1$ injection in the last 4 weeks). ${ }^{15}$ The level of adherence in the population reported here is consistent with these estimates (15 of 55 


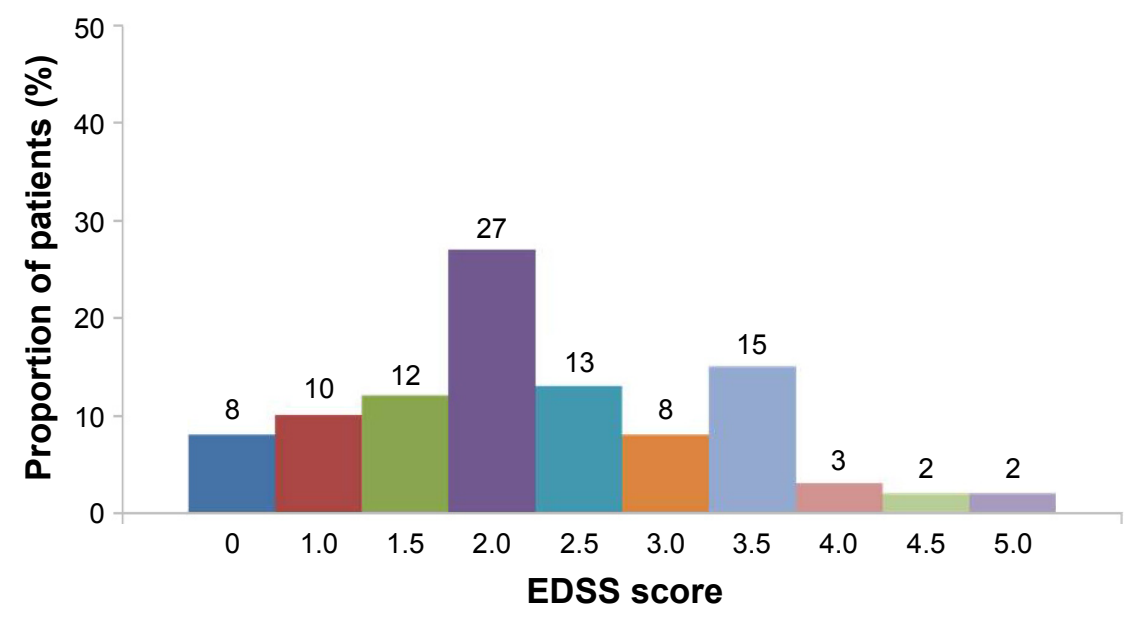

Figure I EDSS score distribution at baseline.

Abbreviation: EDSS, expanded disability status scale.

[27\%] available patients recorded one or more missed dose over the 12 weeks).

The most common reported reasons for nonadherence were forgetting to administer the injection, cited by $50 \%$ of patients, followed by tired of taking injections (20\%), pain at the injection site $(12 \%)$, and injection anxiety $(10 \%) .{ }^{14}$ Other studies have estimated on-treatment injection anxiety to range from $18 \%$ in one longitudinal study ${ }^{16}$ to $22 \%$ in the general population, ${ }^{13}$ and anxiety about injections before starting treatment was expressed by $40 \%$ of patients. ${ }^{16}$ While the use of electronic devices can help to relieve the physical and emotional aspects contributing to lack of adherence by customizing injection parameters, more work will still be required to address the problem of patients forgetting to take their injection. ${ }^{17,18}$

Poor adherence to therapy can result in worse health outcomes..$^{19}$ A retrospective analysis of 2,388 MS patients in the USA identified from a prescription claims database showed that patients who had a gap in treatment that was $\geq 90$ days had a 1.9-fold higher risk of a severe relapse compared with a reference group with a gap in therapy of 0-10 days. ${ }^{19}$ In a separate study comparing patients who were switched from IFN beta- $1 \mathrm{~b}$ three times per week to IFN beta-1a once

Table 3 Adherence to RebiSmart ${ }^{\circledR}$

\begin{tabular}{ll}
\hline Adherence & $\begin{array}{l}\text { Patients, N (\%; exact Clopper- } \\
\text { Pearson 95\% CI) }\end{array}$ \\
\hline$\geq 75 \%$ & $51(94 ; 85-99)$ \\
$\geq 80 \%$ & $50(93 ; 82-98)$ \\
$\geq 85 \%$ & $49(91 ; 80-97)$ \\
$\geq 90 \%$ & $47(87 ; 75-95)$ \\
$\geq 95 \%$ & $41(76 ; 62-87)$ \\
$\geq 100 \%$ & $34(63 ; 49-76)$ \\
\hline
\end{tabular}

per week,,$^{20,21} 77 \%$ of patients who switched experienced a relapse compared with $21 \%$ of patients maintained on treatment three times per week. Furthermore, the importance of adherence with long-term therapy in MS is illustrated by reports that patients discontinuing therapy had a significantly higher EDSS score at follow-up than those who remained on treatment. ${ }^{21}$ Similarly, the risk of disability progression and the worsening of relapse rate were reduced by about four- to fivefold in patients exposed to IFN-beta 1a for more than 4 years compared with patients exposed for up to 2 years. ${ }^{22}$

Overall, these data suggest that a strategy aimed at helping patients remember their dose, improving the comfort of injections, and making injection preparation convenient would help improve treatment compliance. ${ }^{23}$ Some studies ${ }^{12,13}$ have demonstrated that the use of self-injection devices decreases the incidence of injection-site reactions and, most importantly, improves patient compliance with treatment. The incidence of injection-site reactions can be reduced with correct injection technique and by the use of injection devices. ${ }^{17,24}$ However, to date, there has not been a device with the functionality to track injections to provide an accurate estimate of adherence that can be shared between the MS patient and healthcare provider.

The RebiSmart device was developed with the aim of improving adherence by incorporating the following key features: convenience offered by the delivery of multiple fixed doses; a reminder system via the injection log; the ability for patients to customize the settings for injection speed, depth, and duration; detailed step-by-step instructions; and the facility to monitor treatment adherence by tracking of injection history, which can be downloaded, to inform healthcare professionals and motivate patients regarding the level of adherence. 


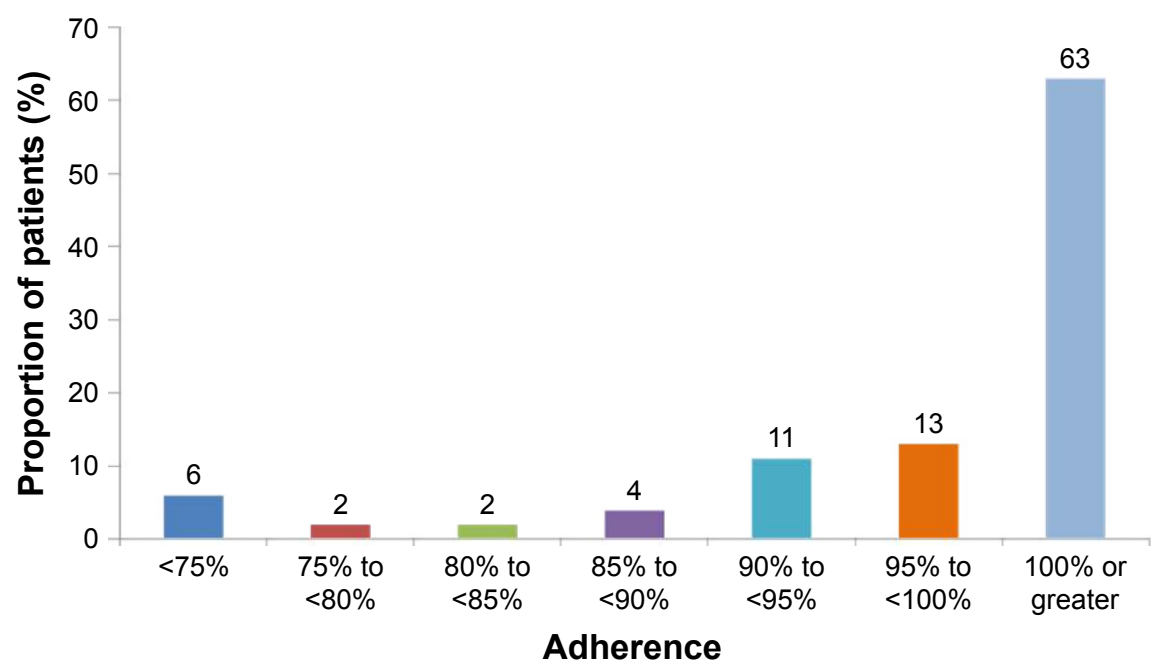

Figure 2 Distribution of patients for each $5 \%$ tier of adherence.

Of the patients using the RebiSmart device, almost $80 \%$ of patients completed more than $90 \%$ of the planned injections over the 12-week period. This high level of adherence with an electronic device is in agreement with the results from other studies. ${ }^{6,25,26}$ Furthermore, although injection-site reactions and influenza-like symptoms were not completely reduced, the treatment was generally well tolerated, and a high proportion of patients expressed satisfaction with the RebiSmart device, largely driven by the convenience it provides. This may indicate that convenience is a key factor in patient adherence to DMDs that could warrant further investigation.

Although the observation period reported here is short, these data indicate that good adherence can be achieved when patients use RebiSmart. In addition to the short time duration, there are other limitations to this study. Difficulty in recruiting patients meant that we were unable to include the estimated 100 patients, and many of the patients included (98\%) had used the RebiSmart device prior to enrolment in the study, therefore, adherence to this device may be better in this group compared with patients with MS in general. Furthermore, as there was no comparator in this single-arm, open-label study, direct comparisons with other injectable DMDs are not possible.

Our results show that the use of the RebiSmart device is potentially important to accurately monitor adherence to treatment in patients with RRMS and may contribute to increased adherence. Almost $80 \%$ of patients stated that "overall convenience" was the most important benefit of the injection system.

\section{Acknowledgments}

The authors would like to thank all the patients who contributed to this study and the investigators at each of the participating clinics, particularly the study nurses. Medical writing assistance in the preparation of this manuscript was provided by Steven Goodrick of Science Communications, London, UK, funded by Merck KGaA, Darmstadt, Germany. This study was sponsored by Merck Norway, an affiliate of Merck KGaA, Darmstadt, Germany.

\section{Author contributions}

EDP was involved in the design of the study; writing of the study protocol; the acquisition and interpretation of the data; the writing, drafting, and reviewing of the manuscript; and the final approval. ES participated in the acquisition and interpretation of data, revising the manuscript, and final approval. JLV, RS, NM, GM, and JLF were involved in the design of the study, the acquisition and interpretation of the data, and the drafting and reviewing of the manuscript. MBJ participated in patient enrolment and data acquisition. All authors are responsible for all aspects of the submitted manuscript. The authors take full responsibility for the data, the analyses and the interpretation, and the conduct of the research; had full access to the data; and had the right to publish any and all data, separate and apart from the attitudes of the sponsor. All authors contributed toward data analysis, drafting and revising the paper and agree to be accountable for all aspects of the work.

\section{Disclosure}

EDP has served on advisory boards for and has received funding for travel related to conferences, meetings, and speaker honoraria from Biogen Idec, Merck, Sanofi-Aventis, Teva, and Novartis. JLV has served on advisory boards for and has received funding for travel related to these activities as well as honoraria from Biogen Idec, Merck, Sanofi-Aventis, Teva, and Novartis. MBJ has received financial compensation for 
participation in advisory boards for TEVA, Novartis, Biogen, and Roche. RS has received financial support for congress participation from several companies. JLF has served on scientific advisory boards and received funding for travel related to these activities as well as honoraria from Biogen Idec, Merck, Sanofi-Aventis, Teva, Novartis, and Almirall. She has received speaker honoraria from Biogen Idec, Teva, and Novartis. GM is an employee of Merck Norway, an affiliate of Merck AB (Merck AB NUF). JLF has served as an advisor on preclinical development for Takeda. NM is an employee of Smerud Medical Research. The authors report no other conflicts of interest in this work.

\section{References}

1. Coles A. Multiple sclerosis. Pract Neurol. 2009;9(2):118-126.

2. Sadovnick AD, Ebers GC, Dyment DA, Risch NJ. Evidence for genetic basis of multiple sclerosis. The Canadian Collaborative Study Group. Lancet. 1996;347(9017):1728-1730.

3. Sadovnick AD, Ebers GC. Epidemiology of multiple sclerosis: a critical overview. Can J Neurol Sci. 1993;20(1):17-29.

4. Katrych O, Simone TM, Azad S, Mousa SA. Disease-modifying agents in the treatment of multiple sclerosis: a review of long-term outcomes. CNS Neurol Disord Drug Targets. 2009;8(6):512-519.

5. Oliver BJ, Kohli E, Kasper LH. Interferon therapy in relapsing-remitting multiple sclerosis: a systematic review and meta-analysis of the comparative trials. J Neurol Sci. 2011;302(1-2):96-105.

6. Devonshire V, Arbizu T, Borre B, et al. Patient-rated suitability of a novel electronic device for self-injection of subcutaneous interferon beta-1a in relapsing multiple sclerosis: an international, single-arm, multicentre, Phase IIIb study. BMC Neurol. 2010;10:28.

7. Menzin J, Caon C, Nichols C, White LA, Friedman M, Pill MW. Narrative review of the literature on adherence to disease-modifying therapies among patients with multiple sclerosis. J Manag Care Pharm. 2013; 19(1 Suppl A):S24-S40.

8. Dor A, Lage MJ, Tarrants ML, Castelli-Haley J. Cost sharing, benefit design, and adherence: the case of multiple sclerosis. Adv Health Econ Health Serv Res. 2010;22:175-193.

9. WHO. Adherence to Long-term Therapies: Evidence for Action; 2003. Available from: http://www.who.int/chp/knowledge/publications/ adherence_report/en/. Accessed March 1, 2018.

10. Costello K, Kennedy P, Scanzillo J. Recognizing nonadherence in patients with multiple sclerosis and maintaining treatment adherence in the long term. Medscape J Med. 2008;10(9):225.

11. Zettl UK, Bauer-Steinhusen U, Glaser T, Hechenbichler K, Limmroth V. Evaluation of an electronic diary for improvement of adherence to interferon beta- $1 \mathrm{~b}$ in patients with multiple sclerosis: design and baseline results of an observational cohort study. BMC Neurol. 2013;13:117.
12. Cramer JA, Cuffel BJ, Divan V, Al-Sabbagh A, Glassman M. Patient satisfaction with an injection device for multiple sclerosis treatment. Acta Neurol Scand. 2006;113(3):156-162.

13. Mikol D, Lopez-Bresnahan M, Taraskiewicz S, Chang P, Rangnow J. A randomized, multicentre, open-label, parallel-group trial of the tolerability of interferon beta-1a (Rebif) administered by autoinjection or manual injection in relapsing-remitting multiple sclerosis. Mult Scler. 2005;11(5):585-591.

14. Devonshire V, Lapierre Y, Macdonell R, et al. The Global Adherence Project (GAP): a multicenter observational study on adherence to disease-modifying therapies in patients with relapsing-remitting multiple sclerosis. Eur J Neurol. 2011;18(1):69-77.

15. Treadaway K, Cutter G, Salter A, et al. Factors that influence adherence with disease-modifying therapy in MS. $J$ Neurol. 2009;256(4): 568-576.

16. Mohr DC, Boudewyn AC, Likosky W, Levine E, Goodkin DE. Injectable medication for the treatment of multiple sclerosis: the influence of self-efficacy expectations and injection anxiety on adherence and ability to self-inject. Ann Behav Med. 2001;23(2):125-132.

17. Bayas A. Improving adherence to injectable disease-modifying drugs in multiple sclerosis. Expert Opin Drug Deliv. 2013;10(3):285-287.

18. Bayas A, Ouallet JC, Kallmann B, Hupperts R, Fulda U, Marhardt K. Adherence to, and effectiveness of, subcutaneous interferon $\beta$-1a administered by RebiSmart ${ }^{\mathbb{}}$ in patients with relapsing multiple sclerosis: results of the 1-year, observational SMART study. Expert Opin Drug Deliv. 2015;12(8):1239-1250.

19. Tan H, Cai Q, Agarwal S, Stephenson JJ, Kamat S. Impact of adherence to disease-modifying therapies on clinical and economic outcomes among patients with multiple sclerosis. Adv Ther. 2011;28(1):51-61.

20. Barbero P, Verdun E, Bergui M, et al. High-dose, frequently administered interferon beta therapy for relapsing-remitting multiple sclerosis must be maintained over the long term: the interferon beta dosereduction study. J Neurol Sci. 2004;222(1-2):13-19.

21. Rio J, Porcel J, Tellez N, et al. Factors related with treatment adherence to interferon beta and glatiramer acetate therapy in multiple sclerosis. Mult Scler. 2005;11:306-309.

22. Trojano M, Russo P, Fuiani A, et al. The Italian Multiple Sclerosis Database Network (MSDN): the risk of worsening according to IFNbeta exposure in multiple sclerosis. Mult Scler. 2006;12:578-585.

23. Saunders C, Caon C, Smrtka J, Shoemaker J. Factors that influence adherence and strategies to maintain adherence to injected therapies for patients with multiple sclerosis. J Neurosci Nurs. 2010;42:S10-18.

24. Lugaresi A. Addressing the need for increased adherence to multiple sclerosis therapy: can delivery technology enhance patient motivation? Expert Opin Drug Deliv. 2009;6:995-1002.

25. Lugaresi A, Florio C, Brescia-Morra V, et al. Patient adherence to and tolerability of self-administered interferon beta-1a using an electronic autoinjection device: a multicentre, open-label, phase IV study. BMC Neurol. 2012;12:7.

26. Wray S, Armstrong R, Herrman C, et al. Results from the single-use autoinjector for self-administration of subcutaneous interferon beta-1a in patients with relapsing multiple sclerosis (MOSAIC) study. Expert Opin Drug Deliv. 2011;8:1543-1553.
Patient Preference and Adherence

\section{Publish your work in this journal}

Patient Preference and Adherence is an international, peer-reviewed, open access journal that focuses on the growing importance of patient preference and adherence throughout the therapeutic continuum. Patient satisfaction, acceptability, quality of life, compliance, persistence and their role in developing new therapeutic modalities and compounds to optimize

\section{Dovepress}

clinical outcomes for existing disease states are major areas of interest for the journal. This journal has been accepted for indexing on PubMed Central. The manuscript management system is completely online and includes a very quick and fair peer-review system, which is all easy to use. Visit http://www. dovepress.com/testimonials.php to read real quotes from published authors. 